\title{
Regional migratory osteoporosis
}

\author{
G.A.C. MAJOR \\ F.R.A.C.P. \\ Department of Rheumatology, Royal Newcastle Hospital, Pacific Street, Newcastle. N.S.W. 2300, Australia
}

\begin{abstract}
Summary
The case history of a patient with regional migratory osteoporosis and associated electromyographic abnormalities is reported.

The changes seen in this patient suggest that radiculopathy secondary to traumatic ischaemia may be the pathogenic basis of this disorder.
\end{abstract}

KEY WORDS: algodystrophy, radiculopathy, traumatic ischaemia.

\section{Introduction}

Regional migratory osteoporosis (RMO) and the related condition of transient osteoporosis of the hips are uncommon disorders which have received relatively scant attention in the literature.

Since the reports by Duncan and his colleagues in 1967 and Lequesne in 1968, drawing attention to these entities several further cases have been reported in the English literature (Hunder and Kelly, 1968a; Rosen, 1970; Swezey, 1970; O'Mara and Pinals, 1970; Pantazopoulos, Exarchou and Hartofilakidis, 1973; Valenzuela, Aris and Jacobelli, 1977; McCord et al., 1978; Tannenbaum et al., 1980) and have shown a consistent clinical pattern, lending support to the original contention that the observed changes constituted a distinct syndrome.

The purpose of this report is to describe a further patient whose disease is illustrative of the syndrome and to report on some associated neuropathic changes which may be of relevance to the pathogenic mechanisms involved.

\section{Case report}

A 53-year-old clerk, presented in August 1979, with a 1-month history of left hip and knee pain, which had developed a week after he had engaged in some strenuous and unaccustomed activity. There was no complaint related to any other peripheral site and he had no back pain. Physical examination was normal apart from some tenderness over the left greater trochanteric bursa. An X-ray of the pelvis and hips was normal (Fig. 1a). He was prescribed indomethacin, which proved to be of marginal help only and the hip pain did not settle until December, at which stage knee pain was still present and an $\mathrm{X}$ ray showed loss of bone density at the knee.

In January 1980 he missed his footing on stepping off a kerb and developed left ankle swelling and pain. The indomethacin was continued and he was referred for physiotherapy. Again the response was poor, and because of persisting problems 5 months later, rheumatology consultation was sought. When seen in June, mild soft tissue thickening and reduced dorsi flexion were noted at the left ankle. The only other abnormal finding was minimal left quadriceps wasting.

$\mathrm{X}$-ray of the ankle showed patchy osteoporosis Full blood count, serum creatinine and electrolyteso serum calcium, alkaline phosphatase, liver function tests and fasting blood sugar, were normal. The ESR was $2 \mathrm{~mm} / \mathrm{hr}$. Rheumatoid factor and antinuclear factor tests were negative. Serum parathyroid hormone was $0.49 \mathrm{mmol} /$ litre (normal range $0-0.4$ mmol/litre).

Non-steroidal anti-inflammatory drug therapy and physiotherapy were continued, but complete abatement of the left ankle involvement did not occur until September.

He remained well over the next 2 months, but in November, after attempting to dig out a tree stump, he developed right-sided low back and buttock pain which over the following 6 weeks became progressively more severe and he began to experience radiating pain and numbness down the right leg in a typical sciatic nerve distribution.

He was seen in the clinic in December, when an absent right ankle jerk and decreased light touch sensation over the lateral aspect of the foot were demonstrated. Straight leg raising was limited to $20^{\circ}$.

$A$ repeat of the earlier haematological and biochemical tests again gave normal results. Plain lumbosacral X-rays showed mild dengenerative changes at L5-S1 apophyseal joints. A metrizamide myelogram was normal. Analysis of a sample of cerebrospinal fluid obtained at myelography, for protein, cells and 


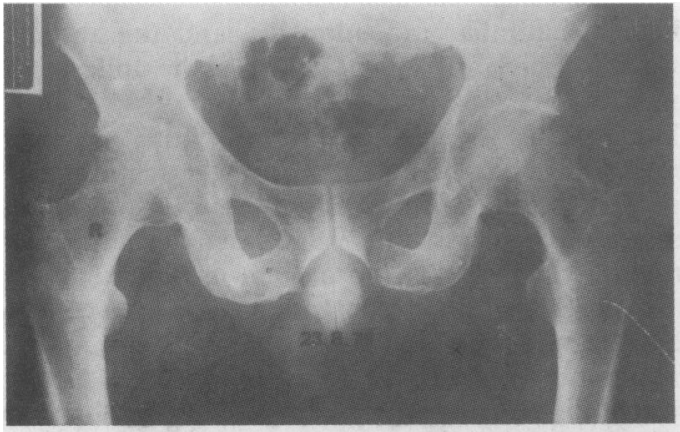

(a)

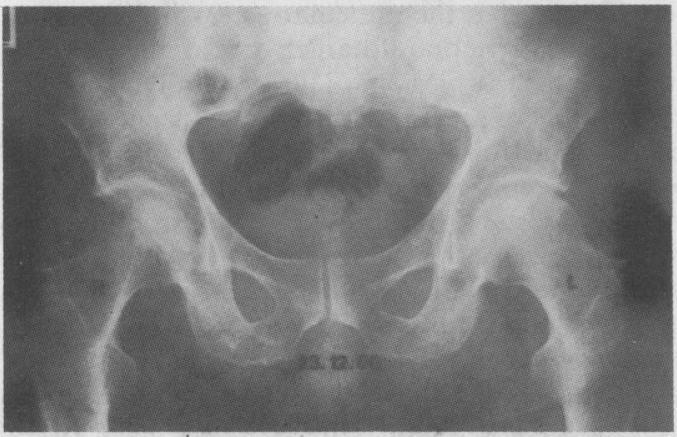

(b)

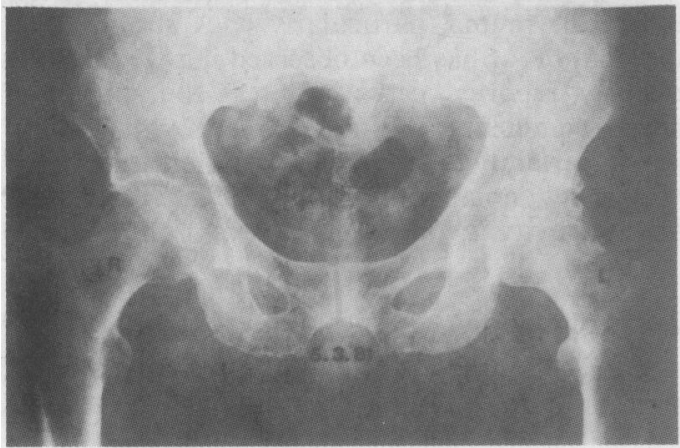

(c)

Fig. 1. (a) Serial X-ray of hips. At presentation-radiologically normal hips. (b) Two months after onset of right hip pain-marked local osteoporosis of right hip, with loss of cortical outline of femoral head. (c) Following clinical recovery, 5 months after onset of symptoms-minimal residual osteoporosis of right hip.

glucose were normal, and the routine culture and syphilis serology were negative.

$\mathrm{X}$-ray of the pelvis and hips showed marked local osteoporosis of the right hip with blurring of the outline of the cortex of the femoral head (Fig. 1b). A bone scan (technetium pyrophosphate) showed marked focal uptake at the right hip (Fig. 2a).

Electromyography (EMG) was performed, and showed an acute denervation pattern, with increased insertional activity, polyphasic units and increased fibrillation in the right gluteus medius, biceps fe-

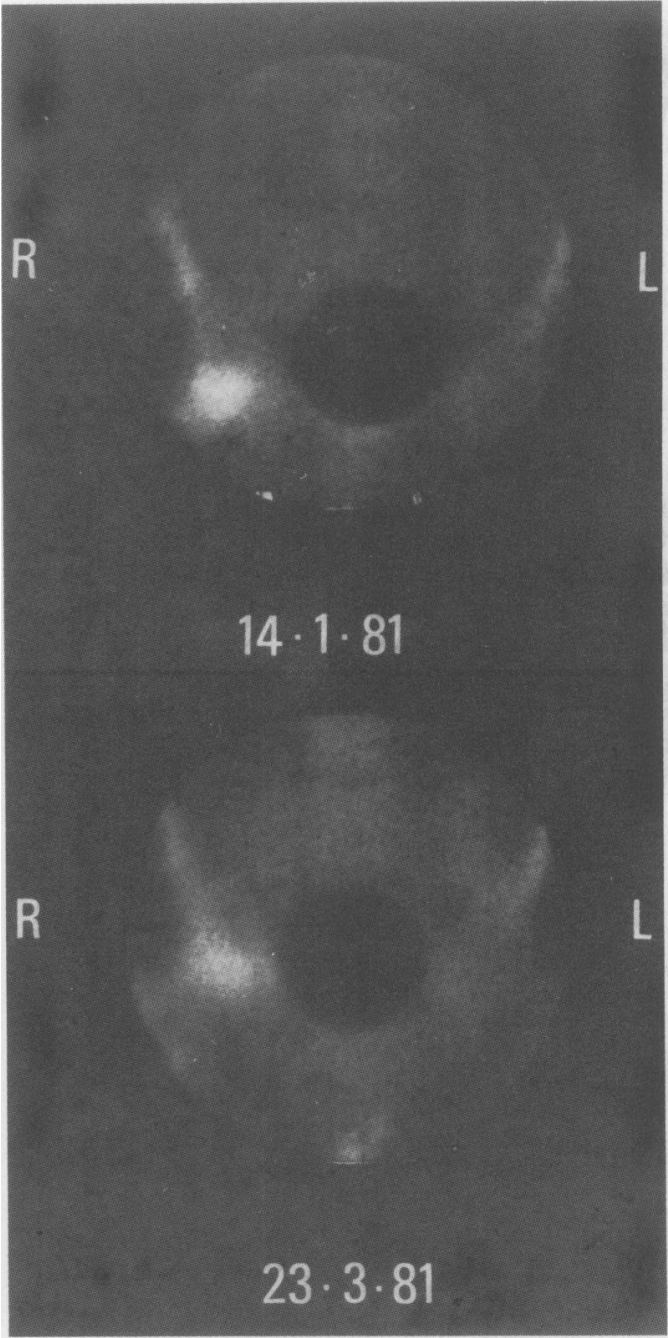

(a)

(b)

FIG. 2. Serial technetium pyrophosphate bone scans. (a) Ten weeks after onset of right hip pain. (b) Following clinical recovery, 5 months after onset of symptoms - some increased uptake of isotope still present, but parallel with the radiological progression, showing evidence of resolution.

moris, vastus lateralis and the right paraspinal muscles (sampled at two sites).

Treatment was with bed rest and, later, assisted weight bearing, non-steroidal anti-inflammatory drugs and physiotherapy.

Gradual symptomatic resolution occurred over a 3-month period. This was associated with distinct improvement in the radiological appearances of the hip (Fig. 1c) and a decrease in the radionucleotide uptake (Fig. 2b). A repeat EMG no longer showed any evidence of active denervation.

A further 60 month follow-up has shown him to 
remain well and without recurrence of joint complaints.

\section{Discussion}

The clinical and radiological features of RMO have been well described in several earlier reports (Duncan et al., 1967; Lequesne, 1968; Hunder and Kelly, 1968a). These features are sufficiently distinctive to allow relatively easy identification, though difficulties may be encountered in the early case, where a relatively wide list of differential possibilities needs to be entertained. The subsequent course, with persistent involvement lasting as a rule several months, and affecting almost exclusively lower limb joints, in the absence of any marked change in acute phase reactants, makes this task much easier. A regularly observed, though unexplained, feature of the syndrome is the lack of recurrence at a site which has been previously involved. The course of the patient reported here (summarized in Fig. 3) is illustrative of these aspects.

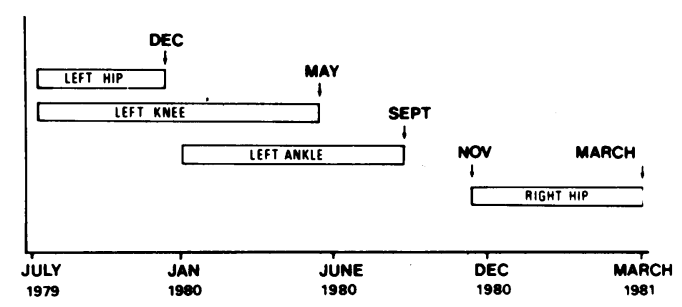

FIG. 3. Summary diagram, showing sequence and pattern of the joint involvement.

Attempts at identifying the pathogenic mechanisms have been generally unsuccessful. Examination of the joint fluid, synovium and bone have consistently failed to show evidence of infection. The histology of the synovium shows an essentially 'noninflammatory' picture, with variable oedema and hypertrophy accompanied by a scanty mononuclear infiltrate only. The bone changes range from osteoporosis to a mixed pattern of osteonecrosis and new bone formation (Duncan et al., 1967; Hunder and Kelly, 1968a; Pantazopoulos et al., 1973; Valenzuela, Aris and Jacobelli, 1977).

Several investigators have commented on the similarities to reflex sympathetic dystrophy (Duncan et al., 1967; Hunder and Kelly, 1968a; Swezey, 1970) and a primary neuropathic mechanism was postulated by Curtiss and Kincaid (1959). However, it was not until the demonstration by McCord and his colleagues of associated EMG abnormalities in a patient with RMO in 1978, that evidence for possible primary neurological lesion emerged. The findings indicated a lumbar radiculopathy and the authors postulated ischaemic vasculopathy as the underlying process.

The changes seen in the present patient confirm these findings and constitute a further such observation. The episode of sciatica, immediately preceding the right hip involvement in this patient, is of interest, particularly in view of the normal myelogram, and lends support to the contention that nerve root ischaemia was the inciting lesion for this episode.

The ventral nerve root receives its blood supply through the anterior radicular branch of the lumbar artery. Trauma to the radicular artery in its passage through the vertebral foramen could theoretically result in such a lesion. The generally observed recovery time of several months is consistent with the time required for nerve regeneration, and suggests that the ischaemia is transient, and that blood supply is readily re-established probably by retrograde flow through the posterior radicular artery which forms free anastomosis with the anterior artery. Such a new course of blood supply which would not be at risk to the same mechanical trauma, provides a potential explanation for the 'lightning-like' behaviour of the syndrome in not striking in the same place twice. Mechanical trauma, particularly back strain, as an antecedent event has been observed in several of the previously reported cases and was the subject of editorial comment by Hunder and Kelly (1968b) This was certainly a feature in this patient and two of the episodes notably consisted of unaccustomed physical activity involving heavy strain on the back.

\section{Acknowledgments}

I would like to thank $\operatorname{Dr} Y$. Ghabrial for his help in the management of this patient, Dr T. Holland for performing the E.M.G. studies, and Miss M. Kidd for kindly providing secretarial assistance.

\section{References}

CURTISS, P.H. \& KINCAID, W.E. (1959) Transitory demineralization of the hip in pregnancy. Journal of Bone and Joint Surgery, 41a, 1327.

Duncan, H., Frame, B., Frost, H. \& ARnstein, A.R. (1967) Migratory osteolysis of the lower extremities. Annals of Internal Medicine, 66, 1165.

Hunder, G.G. \& Kelly, P.J. (1968a) Roentgenologic transient osteoporosis of the hips: A clinical syndrome? Annals of Internal $N$ Medicine, 68, 539.

HUNDER, G.G. \& Kelly, P.J. (1968b) Roentgenologic transient osteoporosis of the hip. Annals of Internal Medicine, 69, 633 (editorial).

LEQUESNE, M. (1968) Transient osteoporosis of the hip. Annals of the Rheumatic Diseases, 27, 463.

MCCORD, W.C., NiES, K.M., CAMPION, D.S. \& Louie, J.S. (1978) Regional migratory osteoporosis: a denervation disease. Arthritis $\mathbb{D}$ and Rheumatism, 21, 834.

O'MARA, R.E. \& PINALS, R.S. (1970) Bone scanning in regional migratory osteoporosis. Radiology, $97,579$. 
Pantazopoulos, T., Exarchou, E. \& Hartofilakidis-GarafALIDIS, G. (1973) Idiopathic transient osteoporosis of the hip. Journal of Bone and Joint Surgery, 55a, 315.

RoSEN, R.A. (1970) Transitory demineralization of the femoral head. Radiology, 94, 509.

SwEZEY, R.L. (1970) Transient osteoporosis of the hip, foot and knee. Arthritis and Rheumatism, 13, 858.
Tannenbaum, H., Esdalle, J. \& Rosenthal, L. (1980) Joint imaging in regional migratory osteoporosis. Journal of Rheumato$\operatorname{logy}, 7,237$.

VAlenzuela, F., ARIS, H. \& Jacobelli, S. (1977) Transient osteoporosis of the hip. Journal of Rheumatology, 4, 59.

(Accepted 12 July 1983) 